\title{
Study on the Effects of IC-ECG Method in Neonatal PICC Catheterization
}

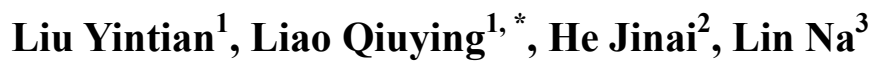 \\ ${ }^{1}$ Department of Cardiovascular Surgery, The First Affiliated Hospital of Jinan University, Guangzhou, China \\ ${ }^{2}$ Department of Nursing, The First Affiliated Hospital of Jinan University, Guangzhou, China \\ ${ }^{3}$ Department of Neonatal Intensive Care Unit, The First Affiliated Hospital of Jinan University, Guangzhou, China \\ Email address: \\ 18928903872@126.com (Liao Qiuying) \\ *Corresponding author
}

\section{To cite this article:}

Liu Yintian, Liao Qiuying, He Jinai, Lin Na. Study on the Effects of IC-ECG Method in Neonatal PICC Catheterization. American Journal of Pediatrics. Vol. 6, No. 2, 2020, pp. 178-182. doi: 10.11648/j.ajp.20200602.31

Received: March 29, 2020; Accepted: April 17, 2020; Published: April 29, 2020

\begin{abstract}
Objective: To compare the effects of Intracavitary Electrocardiography (IC-ECG) method and in vitro measurement for positioning the tip of Peripherally Inserted Central Catheter (PICC) in neonates, and investigate the influence of these two methods on PICC complications. Methods: Neonates who received PICC in the neonate intensive care unit of the First Affiliated Hospital of Jinan University from January 2015 to August 2019 were analyzed retrospectively. Patients were divided into two groups: the observation group with both IC-ECG method and in vitro measurement and the control group only with in vitro measurement. Chest X-ray were taken for both groups after the catheterization to confirm the catheter tip position. Statistics about catheter tip position, final extubation rate and PICC-related complications were collected and compared. Results: A total of 348 patients were enrolled (181 observation group, 167 control group). Two groups were comparable in terms of gender, gestational age, birth weight, catheterized side and vein during catheterization with no statistical significance. In the observation group, $173(95.6 \%)$ had P-wave amplitude changes, including $4(2.2 \%)$ were positioned at the upper $1 / 3$ of the superior vena cava (SVC), $28(15.5 \%)$ at the middle $1 / 3$ of SVC, $104(57.5 \%)$ at lower $1 / 3$ of SVC; 38 cases $(21.0 \%)$ at the right atrium or right ventricle, $7(3.9 \%)$ at other parts, and the proportion of above situations in control group were $7(4.2 \%), 15$ (9.0\%), $33(19.8 \%), 48(28.7 \%)$, and $47(38.3 \%)$, respectively. The final extubation rate in the observation group was $86.2 \%$ $(\mathrm{n}=156)$, and the complication-casued extubation rate was $13.8 \%(\mathrm{n}=25)$ while that in control group was $67.1 \%(\mathrm{n}=112)$ and $32.9 \%(\mathrm{n}=55)$, respectively. The differences between two groups were statistically significant $(P<0.05)$. Conclusion: Application of IC-ECG-guided PICC in neonates can improve the accuracy of catheter tip position and final extubation rate, and reduce the incidence of catheter-related complications.
\end{abstract}

Keywords: Neonate, Intracavitary Electrocardiogram (IC-ECG), Peripherally Inserted Central Catheter (PICC), Catheter Tip Position

\section{Introduction}

IC-ECG method as a technique to locate the proper catheter tip position during PICC catheterization has been recently introduced. A series of multi-center studies have confirmed that the IC-ECG method can be safely applied to adults and children aged from 1 month to 12 years old [1,2]. Later, it has also been proved by some scholars that IC-ECG method can serve as accurate as the chest $\mathrm{x}$-ray in determining the catheter tip position $[3,4]$. Therefore, it has begun to be applied in the neonatal PICC catheterization because of its advantages, such as no radiation and easy operation, but still less studies about it were found.

There are some differences in PICC catheterization between neonates and adults. For example, the PICC catheter for neonates has an open end in a smaller diameter (1.9 Fr); neonates may cry during the operation and therefore affect the process; they have a shorter SVC than that of adults, and show obvious P-wave amplitude increase when approaching the right atrium [5]. In this case, more clinical trials are required to identify whether IC-ECG method can serve efficiently to 
observe the changes of P-wave amplitude during catheterization so as to accurately locate the catheter tip position.

For this purpose, this study retrospectively reviewed the effects of the IC-ECG method and the traditional in vitro measurement method in neonatal PICC, and discussed the feasibility of IC-ECG method. The results are as follow.

\section{Objects and Methods}

\subsection{Study Objects}

Neonates that received PICC catheterization in the neonate intensive care unit of the First Affiliated Hospital of Jinan University from January 2015 to August 2019 were enrolled in this study and divided into two groups. Observation group: both the IC-ECG and in vitro measurement methods were applied. Control group: only in vitro measurement method was adopted to predict the length of PICC before insertion.

Inclusion criteria: neonatal patients with PICC; Exclusion criteria: patients whose catheter tip position was not confirmed by chest X-ray; catheterized patients transferred to other hospitals during treatment; patients failed to insert the PICC.

\subsection{Methods}

\subsubsection{Materials}

Control group: Bard, UTA or Unijet 1.9 Fr open end PICC catheter, PICC puncture medical dressing kit with heparin cap, $10 \mathrm{ml}$ injector, transparent dressing etc.

Observation group: additional support of PHILIPS GS10 ECG monitor, sterilized EKG clip, Braun intracardiac converter, electrode pad and No. 7 scalp needle on the basis of control group.

\subsubsection{Steps of Positioning}

Control group: 1) Predict the insertion length: Place the patient in a supine position and have the lateral puncture extremity stretched $90^{\circ}$, and then take the distance from the puncture point to the right sternoclavicular joint and to the third rib as the insertion length; 2) Fix the catheter when it reaches the predicted length; 3) Take a chest X-ray to confirm the final catheter tip position.

Observation group: 1) Export the surface ECG: First adjust the ECG monitor to lead II, paste 3 electrode pads on the midline of bilateral subclavian bones, the left costal margin and the midine of axillary respectively, then connect the lead wires to export the surface ECG. Next, connect the upper left lead wires to the intracardiac ECG converter and the converter to the ECG monitor; 2) Apply the catheter in the same length as that in control group; 3) IC-ECG method: Connect the catheter with the pre-flushed heparin cap after the catheter is inserted to the predicted length, then insert the scalp needle into the heparin cap and connect the needle to the IC-ECG converter with the sterile ECG clip. Next, turn on the converter. Send in or withdraw the catheter while slowly injecting the saline solution, and observing the $\mathrm{P}$ wave at the same time. Then, withdraw the catheter to a $\mathrm{P} / \mathrm{R}$ amplitude ratio of $50 \%$ to $80 \%$ and fix it when the $\mathrm{P}$ wave reaches the maximal height; 4) Take a chest X-ray to confirm the final catheter tip position.

\subsection{Observation Indicators}

Catheter tip position: 1) For patients with PICC inserted through the veins of upper extremities, such as arms, neck and head: According to the INS guidelines, the most ideal position for the catheter tip is at the lower $1 / 3$ of SVC or CAJ (chest X-ray shows roughly T5-T6 level); proper (ideal) position is at the SVC (chest X-ray shows roughly T2-T6 level); tip position not reaching SVC is considered to be too shallow (chest $\mathrm{X}$-ray shows in the axillary vein, subclavian vein or brachiocephalic vein) while at the right atrium or deeper is too deep [6] (chest X-ray shows roughly below T7 level). 2) For patients with PICC inserted through the veins of lower extremities, such as saphenous vein or femoral vein: tip position at the junction of the inferior vena cava (IVC) and right atrium is considered to be ideal; position below the junction is too shallow while located inside the right atrium is too deep. Positions not mentioned above are considered to be malposition like locating in the internal jugular vein or the azygos vein etc..

Complications: clogging, leakage, secondary catheter malposition caused by growth and development, catheter rupture, infection, allergy, and local swelling.

\subsection{Statistical Methods}

In this study, the results were presented in mean values \pm standard deviation (SD), and analyzed by one sample $t$ test. Data were evaluated by one-way analysis of variance (ANOVA), and the comparison between two groups was carried out by the Tukey HSD post-hoc test with the help of Statistical Package for the Social Sciences ver.24.0 software (SPSS, Chicago, IL). P $<0.05$ was considered statistically significant.

\section{Results}

\subsection{General Information}

Among 411 catheterized neonates in our hospital from January 2015 to August 2019, 348 cases were finally enrolled (181 observation group, 167 control group) with 44 cases screened out. Two groups were comparable in terms of gender, gestational age, birth weight, catheterized side and vein with no statistical significance. $(\mathrm{P}>0.05)$, as shown in Table 1. 
Table 1. General information of the patients.

\begin{tabular}{|c|c|c|c|c|}
\hline Project & Observation group $(\mathrm{n}=181)$ & Control group $(n=167)$ & $\chi^{2} / \mathbf{f}$ & p-value \\
\hline Gender & & & 1.605 & 0.205 \\
\hline male & $115(63.5 \%)$ & $95(56.9 \%)$ & & \\
\hline Female & $66(36.5 \%)$ & $72(43.1 \%)$ & & \\
\hline Intubation limb & & & 2.125 & 0.145 \\
\hline left & $78(43.1 \%)$ & $85(56.9 \%)$ & & \\
\hline right & $103(56.9 \%)$ & $82(49.1 \%)$ & & \\
\hline Catheterization & & & 6.278 & 0.179 \\
\hline Basilic vein & $114(63.0 \%)$ & $95(56.9 \%)$ & & \\
\hline Median cubital vein & $26(14.4 \%)$ & $31(18.6 \%)$ & & \\
\hline Superficial temporal vein & $18(9.9 \%)$ & $25(15.0 \%)$ & & \\
\hline Axillary vein & $17(9.4 \%)$ & $8(4.8 \%)$ & & \\
\hline other & $6(3.3 \%)$ & $8(4.8 \%)$ & & \\
\hline Gestational age (week) & $31.26 \pm 2.18$ & $30.44 \pm 2.22$ & 0.110 & 0.915 \\
\hline body weight $(\mathrm{Kg})$ & $1.54 \pm 0.33$ & $1.30 \pm 0.34$ & 1.277 & 0.259 \\
\hline
\end{tabular}

\subsection{Comparison of the P-wave Amplitude Change and Tip Position Between Two Groups}

Among all 181 cases in the observation group, 173 cases had changes in P-wave amplitude during PICC catheterization while the rest of them showed no evidence of that. The P-wave amplitude changes of the observation group are shown in Table 2. The comparison of catheter tip position between the two groups is shown in Tables 3 and 4 . The differences between the two groups were statistically significant $(\mathrm{P}<0.05)$.

Table 2. Changes of P-wave amplitude and catheter tip position in the observation group.

\begin{tabular}{|c|c|c|c|c|c|}
\hline \multirow{2}{*}{$\begin{array}{l}\text { Whether p-wave amplitude } \\
\text { changes }\end{array}$} & \multicolumn{4}{|c|}{ Catheter tip position } & \multirow{2}{*}{$\begin{array}{l}\text { Total } \\
(n=181)\end{array}$} \\
\hline & SVC & RA & Not reaching SVC & Malposition & \\
\hline appear & 136 & 37 & - & - & 173 \\
\hline not appear & - & 1 & 5 & 2 & 8 \\
\hline
\end{tabular}

Table 3. Comparison of the catheter tip position between the two groups.

\begin{tabular}{lllll}
\hline \multirow{2}{*}{ Group } & \multicolumn{2}{l}{ Catheter tip position } & & Malposition \\
\cline { 2 - 5 } & SVC & Not reaching SVC & RA & 2 \\
\hline observation group $(\mathrm{n}=181)$ & 136 & 5 & 38 & 9 \\
control group $(\mathrm{n}=167)$ & 69 & 41 & 48 & \\
$\chi^{2}$ value & 55.215 & & & \\
p-value & 0.01 & & & \\
\hline
\end{tabular}

Table 4. The position of the two groups in SVC.

\begin{tabular}{lll}
\hline \multirow{2}{*}{ Group } & Superior vena cava & \\
\cline { 2 - 3 } & Ideal position & Non-ideal position \\
\hline observation group $(\mathrm{n}=136)$ & 105 & 31 \\
control group $(\mathrm{n}=69)$ & 32 & 37 \\
$\chi^{2}$ value & 10.626 & \\
p-value & 0.01 & \\
\hline
\end{tabular}

\subsection{Comparison of Treatment Outcomes and the Incidence of Complications Between the Two Groups}

Outcomes and incidence of complications of the two groups are compared in Tables 5 and 6 , which were both statistically significant $(\mathrm{P}<0.05)$.

Table 5. Comparison of treatment outcomes between the two groups.

\begin{tabular}{lll}
\hline \multirow{2}{*}{ Group } & Outcomes & \\
\cline { 2 - 3 } & Extubation after treatment & Complication-caused Extubation \\
\hline observation group $(\mathrm{n}=181)$ & 156 & 25 \\
control group $(\mathrm{n}=167)$ & 112 & 55 \\
$\chi^{2}$ value & 17.940 & \\
$p$-value & 0.01 & \\
\hline
\end{tabular}


Table 6. Comparison of complications during catheterization between the two groups.

\begin{tabular}{llll}
\hline \multirow{2}{*}{ Group } & Complications & & 0thers * \\
\cline { 2 - 4 } & Clogging & Leakage & 3 \\
\hline observation group $(\mathrm{n}=25)$ & 19 & 3 & 24 \\
control group $(\mathrm{n}=55)$ & 19 & & 12 \\
$\chi^{2}$ value & 12.199 & & \\
$p$-value & 0.002 & & \\
\hline
\end{tabular}

Others*: include secondary catheter malposition due to growth and development, catheter rupture, infection, allergy and local swelling.

\section{Discussion}

\subsection{Significance of IC-ECG Method in Improving the Accuracy of Catheter Tip Position}

In the observation group, $173(95.6 \%)$ cases of neonates had P-wave amplitude changes and $8(4.4 \%)$ cases did not. According to the results of chest X-ray, the ideal tip position rate in the observation group was $57.5 \%(104 / 181)$, which was significantly higher than that of the control group $(19.8 \%$ $(33 / 167))$. In recent years, IC-ECG method has more fully developed in adult PICC catheterization. Foreign studies have reported that the ideal tip position rate can reach $65 \%$ (66/102) [7] among adults, and a series of domestic studies have also shown ideal rate ranging from $38.3 \%(23 / 60)$ [8] to $81.3 \%(1294 / 1592)$ [9].

Researches on the application of IC-ECG method in neonatal PICC have shown ideal position rate ranging from $67.9 \%(19 / 28)$ [8] to $74.7 \%(59 / 79)$ [9] while that in this study was lower than the above figures with $20.4 \%(37 / 181)$ cases inserted to the right atrium or right ventricle (non-ideal position) in the observation group, which might be the results of setting the reference value of $\mathrm{P} / \mathrm{R}$ amplitude ratio in the range of $50-80 \%$ during operation as chest X-ray showed that the catheter tip had entered the right atrium when the $P / R$ ratio was within the range of $70 \%-80 \%$ in some patients.

P-wave, also called the atrial depolarization wave, is the potential change generated during atrial depolarization [10]. Its waveform and amplitude reflect the distance and relative position between the probe and the atrial comprehensive vector axis [11]. The IC-ECG method is to convert the body surface ECG into the IC-ECG. Real time catheter tip position is determined through the changes of P-wave amplitude: the saline solution injected during catheterization work as the electrode, and the $\mathrm{P}$ wave amplitude increases once the catheter tip enters SVC and reaches the peak when arrives at the CAJ, and a P-wave inversion occurs when enters the right atrium. In this study, 8 cases had no detection of $\mathrm{P}$ wave during the PICC catheterization among which 4 cases were caused by catheter length miscalculation (too short), 1 case was interfered by the ventilator, and 3 cases still had no evidence of P-wave after reposition.

This research showed that when the the catheter tip was more likely to be located in the SVC when the P-wave amplitude changes frequently but there were still $21.0 \%$ cases catheterized in deep position, indicating that the changes of P-wave amplitude change was an important indicator to identify whether the catheter entered the SVC but its role in final tip position determination is to be discussed.

\subsection{Significance of IC-ECG Method in Reducing PICC-related Complications}

In this study, $25(13.8 \%)$ cases in the observation group have complications, which was significantly less than that of 55 $(32.9 \%)$ in the control group. The differences were statistically significant. Poor catheter tip location was the key factor that caused catheter-related complications and reduced the service life of the catheter [12]. Excessively deep insertion may cause arrhythmia and pericardial tamponade [13], increased cardiac load, cardiomegaly and even heart failure. Excessively shallow insertion (e.g. the catheter tip is located in the subclavian vein where has narrow blood vessel) may cause the catheter to easily have friction with the blood vessel wall, and the liquid constantly stimulates the blood vessel, resulting in complications like fluid extravasation, phlebitis, and fibrin sheath misalignment [13]. Malposition of catheter tip may result in complications like exudation at the puncture point and limb swelling [14]. Secondary position change takes place as the neonates grow, and when the catheter tip gradually moves up and out of the SVC, the incidence of catheter-related complications increases and the service life of the catheter may be shortened. Therefore, placing the neonatal PICC at the optimal position can eliminate the adverse effects brought by the growth of neonates and is particularly important for completing the entire treatment.

\subsection{Safety Analysis of IC-ECG Method}

No evidence of IC-ECG-related complications (e.g arrhythmia) were found in this study.

\section{Conclusion}

The IC-ECG method can be safely applied in neonatal PICC to increase the accuracy of catheter tip position and reduce the risks of related complications. The P-wave amplitude change during the catheterization is an important indicator to identify whether the catheter tip enters the SVC but whether IC-ECG method can help determine the optimal position of the catheter tip is still required more clinical trials.

\section{References}

[1] Pittiruti M, Bertollo D, Briglia E, et al. The intracavitary ECG method for positioning the tip of central venous catheters: results of an Italian multicenter study. Journal of Vascular Access. 2012; 13 (3): 357. 
[2] Rossetti F, Pittiruti M, Lamperti M, Graziano U, Celentano D, Capozzoli G. The intracavitary ECG method for positioning the tip of central venous access devices in pediatric patients: results of an Italian multicenter study. Journal of Vascular Access. 2014; 13 (3): 1-9.

[3] Wang Huaping, Yang Lixia, Shi Lan, et al. Application of intraluminal electrocardiogram combined with ultrasound in PICC localization. Nursing Practice and Research. 2016; 13 (04): 114-116.

[4] Yuan L, Li R, Meng A, et al. Superior success rate of intracavitary electrocardiogram guidance for peripherally inserted central catheter placement in patients with cancer: A randomized open-label controlled multicenter study. PLoS One. 2017; 12 (3): e0171630.

[5] Li Li, Pang BAodong, Li Yingyu, et al. Characteristics of right heart parameters in electrocardiogram of newborns with different gestational ages. China Journal of Modern Medicine. 2013 (25): 47-50.

[6] Yuan Baoyu, Zheng zheng, Xia Dongmei. Discussion on the improvement method and ideal depth of PICC tube placement. Chinese Journal of General Surgery (Electronic Edition). 2011; 5 (03): 265-266.

[7] Cales YK, Rheingans J, Steves J, Moretti M. Electrocardiogram-Guided Peripherally Inserted Central Catheter Tip Confirmation Using a Standard Electrocardiogram Machine and a Wide-Mouth Electrocardiogram Clip Compared with Traditional Chest
Radiograph. Journal of the Association for Vascular Access. 2016; 21 (1): 44-54.

[8] Wu Wenli, Chen Peiyi, Song Yanling, et al. Observation on The Effect of Intracavity Electrocardiogram Technology on Head-end Positioning of PICC Catheterization in Neonates. The Journal of Practical Medicine. 2016; 32 (20): 3443-3445.

[9] Zhou L, Xua H, Xu M, Hu Y, Lou XF. An Accuracy Study of the Intracavitary Electrocardiogram (IC-ECG) Guided Peripherally Inserted Central Catheter Tip Placement among Neonates. Open Med. 2017; 12 (1): 125-130.

[10] Lu Xilie. 301 Clinical Electrocardiography. Beijing: Science and Technology Literature Press, 2010.

[11] Li Jianguo, Du Chaohui, Zhou Qing, et al. Endocavitary Electrocardiography Guided Cannulation in Placement in Placement of Central Venous Catheters. Chinese Journal of Clinical Nutrition. 2005 (01): 30-34.

[12] Gao Ling. Study for The Relationship Befaeen The Tip of PICC Position and Complication. Journal of Nurses Training. 2012; 27 (22): 2074-2075.

[13] $\mathrm{Wu}$ Xuhong. The Risk Factors and Nursing Intervention Method Related to PICC Complications of Newborn Baibes. Chinese Nursing Management. 2017; 17 (02): 166-171.

[14] Lou Xiaofang, Lu Hua, Li Lizhong, et al. Analysis on reason of abnormal catheter removal of PICC during NICU. Journal of Nurses Training. 2004 (09): 776-777. 